10 of which are $\operatorname{COS} \mathrm{B}$ discoveries.

Two sources, the Crab and Vela, are known pulsars in supernovae remnants, one is possibly the strong X-ray source Cyg X-3, but the other 10 have either a weak or no association with soft $\mathrm{X}$-ray sources or radio pulsars. They may be point-like or emission regions up to $2^{\circ}$ wide. One may be associated with a source of hard $\mathrm{X}$ rays seen by Ariel 5 (Coe et al. IAU Circ. No. 3003, 1976). Hermsen and colleagues stress that the $\gamma$-ray sources contribute significantly to the overall $\gamma$-ray luminosity of the Galaxy. Indeed, as pointed out by Strong et al. (12th ESLAB Symposium, Frascati, 1977), removal of point sources leaves very little evidence for a diffuse galactic emission from and beyond the Perseus arm, just outside $10 \mathrm{kpc}$ from the galactic centre. It is tempting to conclude from the much increased fine structure seen in the $\operatorname{Cos}$ B $\gamma$-ray intensity distribution that point source emission dominates and that little information on glactic cosmic ray motion can be gained by study of the disk photon emission.

Attacking the problem from the other end of the spectrum, the
Imperial College group have suggested that at least 8 (maybe $>16$ ) galactic hard X-ray sources may plausibly be extrapolated into the $\gamma$-ray region to account for a significant amount of the local $\gamma$-ray intensity (Plovdiv C.R. Conf. 1, 152; 1977). However, conventional accretion models for X-ray sources involving essentially thermal heating processes have difficulty in explaining $\gamma$-ray emission and a young pulsar model requiring electromagnetic acceleration would seem to be necessary, as invoked by Lamb et al. (Astrophys. J. 212, L63; 1977) to $\mathrm{ex}^{-}$ plain possible $\gamma$-ray production in Cyg $\mathrm{X}-3$.

Until the contribution of point sources to the galactic disk emission is resolved, $\gamma$-ray astronomers cannot know whether they are chiefly contributing to knowledge of cosmic ray motion in the Galaxy or studying an important class of high energy photon sources involving non-thermal particle acceleration. Better angular resolution and sensitivity throughout the hard $\mathrm{X}$-ray and $\gamma$-ray detector range is required to resolve this particular problem.

\title{
Polarisation in quasars
}

\section{from F. Graham Smith}

THE famous quasar 3C 273, which was the first to be shown to be both a radio source and an optical object with large red-shift, has been studied by every conceivable technique at radio, infrared, optical and X-ray wavelengths. It has spatial structure, a complex spectrum and polarisation, all of which vary with time scales between days and years. The physical processes behind these variations are practically unknown, and observers must sometimes despair of the possiblity of making the key observation which will lead to an unravelling of the complex knot of behaviour. The paper from the Crimean Observatory (in this issue of Nature, page 493) puts a little order into the great variety of behaviour by showing that some of the radio and optical variations are related. The work has involved measurements of polarisation, both optical and radio, which have been difficult to make and which were for some time in doubt.

Variations in radio flux from 3 C 273 were well known in 1966 . They are most marked at short wavelengths: at $40 \mathrm{~cm}$ not much happens, but at $2 \mathrm{~cm}$ the flux density varies by a large factor. The spectra of several quasars

F. Graham Smith is Director of the Royal Greenwich Observatory. show these variations, which indicate an activity close to the core of the quasar, giving a rapid rise in radio emission first at short wavelengths and later, with less intensity, at longer wavelengths. Aller and Haddock at Michigan found that these variations were accompanied by changes in radio polarisation.

Optical polarisation was naturally searched for at this time, and indeed there was already a positive result by W. Liller in 1963, who reported verbally to the American Astronomical Society. Appenzeller concluded in 1968 that there was $0.26 \%$ linear polarisation, probably of interstellar origin. Results from the $2.6 \mathrm{~m}$ Crimean telescope then suggested the existence of circular polarisation, but at such a low level that the techniques were questionable. The observations were made at the coudé focus after several reflections, which easily create spurious polarisation. In 1972 these observations were restarted at the Cassegrain focus, with a more symmetrical optical system and checks with stars with well established circular polarisation showed a measuring accuracy of $0.01 \%$. Both radio and optical observations of polarisation are achieving truly remarkable sensitivity.
3C 273 has an optical jet, a detached radio source $(\mathrm{A})$ and a central source in which the radio object 3 C $273 \mathrm{~B}$ has at least three components with milliarcs diameters. Recent long baseline inteferometer measurements (Legg et al. Astrophys. J. 211, 21; 1977) show that the radio variability is concentrated in one of these three components, on the NE (anti-jet) side of the nucleus. Assuming that the radio emission is synchroton, the magnetic field in this component appears to be about $10^{-2}$ gauss $\left(10^{-6} \mathrm{~T}\right)$. This field is very low in comparison with the stellar surface fields usually responsible for circular polarisation and it seems unlikely that this variable radio source can be the origin of circularly polarised optical emission. This must instead be related to the nucleus where much stronger magnetic fields might be encountered. Presumably the linear polarisation which is now observed to vary also originates in the nucleus.

The interesting aspects of the observations now reported are the rapidity of the variations both at radio and optical wavelengths, and the connection they seem to give between the NE radio component and the nucleus. If the results can be confirmed they should give a more coherent picture of the way in which the nucleus is pumping energy into the outlying sources, which is a crucial question in the origin of the extended radio sources frequently associated with quasars and radio galaxies.

\section{Electrophysiology of the hypothalamus}

from J. L. Barker

A symposium on the Electrophysiology of the Hypothalamus was held at Hemingford Grey House in Cambridge on 24-26 July in conjunction with the 27th International Physiological Congress in Paris. It was organised by R. Dyer (ARC Cambridge) and R. Dyball (ARC Cambridge).

THE hypothalamus is associated with many bodily functions including autonomic reflexes, feeding and sexual behaviour, neuroendocrine control of anterior pituitary activity, thermoregulation and probably, metamor-

J. L. Barker is in the National Institute of Neurological and Communicative Diseases and Stroke, National Institutes of Health, Bethesda, Maryland. 